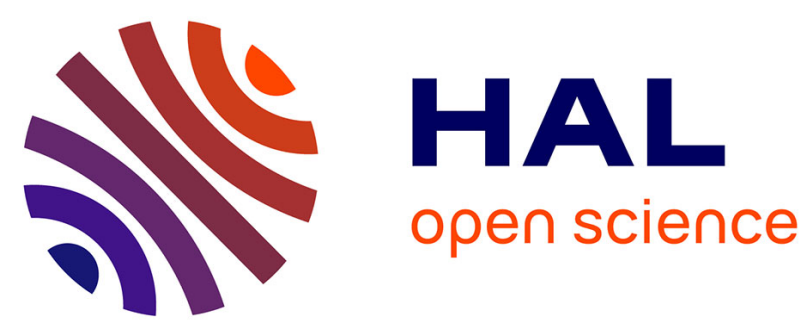

\title{
Picosecond and sub-picosecond pulse generation in semiconductor lasers
}

\author{
J.-M. Lourtioz, Laurent Chusseau, N. Stelmakh
}

\section{To cite this version:}

J.-M. Lourtioz, Laurent Chusseau, N. Stelmakh. Picosecond and sub-picosecond pulse generation in semiconductor lasers. Journal de Physique III, 1992, 2 (9), pp.1673-1690. 10.1051/jp3:1992204 . jpa-00248835

\section{HAL Id: jpa-00248835 https://hal.science/jpa-00248835}

Submitted on 1 Jan 1992

HAL is a multi-disciplinary open access archive for the deposit and dissemination of scientific research documents, whether they are published or not. The documents may come from teaching and research institutions in France or abroad, or from public or private research centers.
L'archive ouverte pluridisciplinaire HAL, est destinée au dépôt et à la diffusion de documents scientifiques de niveau recherche, publiés ou non, émanant des établissements d'enseignement et de recherche français ou étrangers, des laboratoires publics ou privés. 
Classification

Physics Abstracts

$42.55 \mathrm{P}-42.60 \mathrm{~F}-42.80 \mathrm{~W}$

\title{
Picosecond and sub-picosecond pulse generation in semiconductor lasers
}

\author{
J.-M. Lourtioz, L. Chusseau and N. Stelmakh \\ Institut d'Electronique Fondamentale, URA 22 du CNRS, Université Paris XI, Bât. 220, \\ 91405 Orsay Cedex, France
}

(Received 5 December 1991, accepted 20 February 1992)

\begin{abstract}
Résumé. - La génération d'impulsions courtes dans les lasers à semiconducteurs est d'intérêt pratique pour un nombre croissant d'applications. Elle présente aussi un intérêt fondamental de par sa relation étroite avec les études de la dynamique du milieu semiconducteur. Après une revue introductive, nous comparons les performances des techniques de commutation de gain, de commutation de pertes de cavité et de blocage de modes. La comparaison est aussi bien illustrée par des résultats expérimentaux que par des résultats de modélisation. Certains de ces résultats sont nouveaux. Un accent particulier est mis sur les aspects de modulation de phase dans le milieu semiconducteur. Nous discutons finalement quelques perspectives de développements nouveaux.

Abstract. - Generation of short optical pulses in semiconductor lasers is of practical interest for an increasing number of applications. It is also of fundamental interest because of its tight relation with the field of semiconductor laser dynamics. After an introductory review, we compare the performances of gain-switching, Q-switching and mode-locking techniques. The comparison is illustrated by experimental results as well as by laser modelling, some of which are new. A special emphasis is given on the aspects of phase modulation in the semiconductor laser medium. Finally, we discuss some perspectives for further developments.
\end{abstract}

\section{Introductory review.}

High-speed semiconductor light sources generating short pulses in the picosecond and subpicosecond time scales are essential for a large variety of applications. To date, the strongest demand for picosecond laser diodes remains in the field of optical communications where exists a need of sources for soliton transmission in optical fibers [1-3] as well as for high bit rate externally modulated transmission systems and for time-division multiplexed transmission systems $[4,5]$. (Sub)-picosecond laser diodes are also expected to serve as clock generators in all-optical parallel processors. On the other hand, high-power pulsed semiconductor lasers are an interesting alternative to more conventional laser systems of larger sizes (e.g. YAG, dye and solid-state lasers) for electro-optic sampling [6,7], time-resolved spectroscopy, laser ranging and metrology in the time-domain. In addition to their compactness, semiconductor lasers have the evident advantage of being compatible with integrated electronic circuits. 
Different pulse characteristics are desired according to the application which is envisaged. For instance, long-distance soliton transmission in fibers require Fourier transform-limited pulses of adjustable length, while studies of nonlinear phenomena in various materials essentially need high peaks powers. For each application, different techniques and optical schemes must be explored. As for other lasers, there are basically three techniques for operating semiconductor lasers in pulsed regime : i) gain-switching (i.e. pulsed pumping), ii) Q-switching, iii) mode-locking. These three techniques were investigated early on in the development of semiconductor lasers. Moreover, the first laser emission from an homojunction diode structure was achieved in 1962 with the injection of current pulses [8]. Two years later, Lasher proposed an inhomogeneous pumping of the active medium to realize the conditions for regularly pulsed emission [9]. Q-switching was effectively demonstrated in 1966 by Kurnosov et al. with a two-section laser geometry [10]. Mode synchronisation in an injection semiconductor GaAs laser with an external cavity was claimed by Morozov et al. in 1968 [11]. All these first experimental investigations were performed with homojunction structures operated at a low temperature below $77 \mathrm{~K}$. The shortest pulses were already found to be less than $100 \mathrm{ps,}$, with pulse repetition rates of several gigahertz [12].

Progress accomplished in the following decade obviously followed the technological developments of semiconductor materials. Better accuracy was also achieved in pulse measurements. In 1970, E. P. Harris clearly demonstrated active mode-locking in a semiconductor laser [13]. In the same year, Glodge and Lee reported accurate measurement of Q-switched GaAs laser pulses by autocorrelation [14]. After a long period devoted to the search for new lasing materials as well as to the improvement of CW laser spectral properties, P. T. Ho et al. reported in 1978 the first mode-locking experiments with a doubleheterostructure laser operated at room temperature [15]:20 ps pulses were obtained. Passive mode-locking with a saturable absorber was demonstrated three years after by Van der Ziel et al. [16] : the absorber was created by proton implantation through the diode facets. For the first time, subpicosecond pulses $(0.65 \mathrm{ps})$ were measured in semiconductor lasers. This was followed by the work of Yokoyama et al., where similar performances were achieved (0.58 ps) with an aged laser [17]. Gain-switching experiments in double heterostructure GaAs lasers were carried out in parallel, which gave output pulses of $15 \mathrm{ps}$ [18]. All these experimental results represented decisive improvements in terms of short pulse duration.

From the year 1982, the research work was pursued in new directions. The main direction was certainly the investigation of chirping effects in modulated and pulsed lasers with the goal of realizing coherent emission for optical communication [19-21]. These investigations were carried out in parallel with the development of single-mode distributed-feedback (DFB) InGaAsP lasers emitting in spectral regions near $1.3 \mu \mathrm{m}$ and $1.5 \mu \mathrm{m}$. In 1986, Takada et al. reported transform-limited picosecond pulse generation at $1.3 \mu \mathrm{m}$ by fiber compression of gain-switched DFB laser pulses [22]. The efficiency of this novel technique was further confirmed by several authors for the spectral region near $1.5 \mu \mathrm{m}[23,24]$. Fiber compression was also successfully applied to a single-mode Q-switched laser where strong chirping effects led to the generation of very short $(\sim 1 \mathrm{ps)}$ and powerful $(3 \mathrm{~W})$ pulses [25]. Other compression schemes based on gratings and interferometers were used to compensate for chirping effects in mode-locked lasers. Transform-limited pulses were obtained by Silberberg et al. [26] and Kuhl et al. [27] using passive and active mode-locking, respectively.

A second important improvement of (sub)-picsecond laser diodes during the last decade concerned the increase in pulse repetition rate. For gain-switching as well as for active modelocking, progress resulted from the use of lasers with large modulation bandwidths. A repetition rate of $12 \mathrm{GHz}$ was achieved with a gain-switched DFB laser in 1986 [22]. Pulses repetition rates scaling from $16 \mathrm{GHz}$ to $40 \mathrm{GHz}$ were reported in different works on active 
mode-locking [28-31], the highest performance being achieved in 1989 by Tucker et al. with a monolithic extended cavity InGaAsP laser [31]. More recently, passive mode-locking was demonstrated in lasers without extended cavities $[32,34]$, leading to ultimate repetition rates up to $350 \mathrm{GHz}$ [34].

Finally, an increasing number of studies was directed at peak-power optimization. In a few experiments, peak powers exceeding $1 \mathrm{~W}$ were directly obtained from solitary diodes [25, 3537]. However, in most cases, high-power performances were attained by complementing the laser diode with several amplifier sections and nonlinear compression stages. A peak power of $70 \mathrm{~W}$ was reported by Delfyett et al. for an elaborate system comprised of a mode-locked AlGaAs laser diode with an external cavity, a grating compressor and two travelling-wave semiconductor amplifiers [38]. Peak powers scaling from $100 \mathrm{~W}$ to $1 \mathrm{~kW}$ were achieved in two recent experiments by using high-order soliton effects in fiber compression stages : the laser source was a gain-switched DFB laser in one case [39] and a synchronously pumped surfaceemitting InGaAs laser in the other case [40]. Extremely short pulse durations of $0.46 \mathrm{ps,}$ $0.2 \mathrm{ps}$ and $21 \mathrm{fs}$ were reported in references [38, 39] and [40] respectively, but the semiconductor laser characteristics were for a little in these performances.

Following this introductory review, the rest of the paper is organized in four sections. The three techniques for short pulse generation, i.e., gain-switching, Q-switching and modelocking, are presented in sections 2, 3 and 4, respectively. Experimental results and calculations from laser modelling are used to illustrate these techniques. Special emphasis is given on the aspects of phase modulation (chirping) which are of prime importance in semiconductor lasers. We finally discuss some perspectives of further developments in section 5 .

\section{Gain-switching.}

Gain-switching is achieved with a fast excitation of the laser medium. Under this condition, strong population inversion and thus high gain level can be reached before the laser starts. In turn, as soon as lasing takes places, the emitted light is very intense and the populations of the upper laser level rapidly decrease below threshold. The laser emission then ceases and an optical pulse is obtained. Semiconductor lasers are characterized by high gain values and short cavity roundtrip times $(\leqslant 10 \mathrm{ps})$. These two features give the possibility of short pulse generation at high repetition rates by gain-switching.

The simplest mathematical description of the gain-switching process is provided by the two familiar rate equations for the carrier density $N$ and the photon density $S$ :

$$
\begin{aligned}
\frac{\mathrm{d} N}{\mathrm{~d} t} & =\frac{I}{e V}-\frac{N}{\tau_{\mathrm{n}}}-A(1-\varepsilon S)\left(N-N_{0}\right) S \\
\frac{\mathrm{d} S}{\mathrm{~d} t} & =-\frac{S}{\tau_{\mathrm{p}}}+\frac{\Gamma \beta N}{\tau_{\mathrm{n}}}+\Gamma A(1-\varepsilon S)\left(N-N_{0}\right) S .
\end{aligned}
$$

The three terms on the right-hand side of equation (1) represent the carrier injection rate, the spontaneous recombination rate and the stimulated recombination rate, respectively. Those on the right-hand side of equation (2) are respectively the total cavity-loss rate, the spontaneous-emission rate and the stimulated-emission rate. Parameters entering equations (1) and (2) are listed in table I.

Exact solutions of equations (1) and (2) can only be found numerically. Results of numerical simulations are shown in figures 1 and 2 for particular cases of excitation. The first case (Fig. 1a) corresponds to an ideal step excitation with an infinitely short risetime. If the step amplitude exceeds threshold current, the laser output exhibits relaxation oscillations before reaching a steady-state value different from zero. Actually, the laser response is that of 
Table I. - List of parameters entering into rate equations (left) ; parameter values used for calculations in figures 1,2 and 3 (right).

\begin{tabular}{|ll|c|}
\hline \multicolumn{1}{|c|}{ Parameters } & $\begin{array}{c}1.5 \mu \mathrm{m} \text { DFB laser } \\
(L=400 \mu \mathrm{m})\end{array}$ \\
\hline$V$ & active volume & $170 \mu \mathrm{m}^{3}$ \\
$\tau_{\mathrm{n}}$ & spontaneous carrier lifetime & $1 \mathrm{~ns}$ \\
$\tau_{\mathrm{p}}$ & photon lifetime & $1 \mathrm{ps}$ \\
$A$ & linear gain coefficient & $2 \times 10^{-6} \mathrm{~cm}^{3} / \mathrm{s}$ \\
$\varepsilon$ & nonlinear gain coefficient & $1 \times 10^{-17} \mathrm{~cm}^{3}$ \\
$N_{0}$ & carrier density at transparency & $1 \times 10^{18} \mathrm{~cm}^{-3}$ \\
$\Gamma$ & confinement factor & 0.5 \\
$\beta$ & spontaneous emission factor & $2 \times 10^{-5}$ \\
$\alpha$ & linewidth enhancement factor & 4 \\
$\eta$ & laser quantum efficiency & 0.4 \\
\hline
\end{tabular}

a second-order filter whose time evolution is governed by two differential equations. The second case (Fig. 1b) corresponds to a rectangular pulse excitation. A single gain-switched pulse is emitted when the back edge of the current pulse nearly coincides with the end of the first relaxation oscillation. The last case (Fig. 2) corresponds to a strong microwave modulation of the injection current, this situation being of practical interest for high-bit-rate optical communication. Figures $2 a, 2 b$ and $2 c$ represent the simultaneous time-evolutions of the gain, output power and laser frequency, respectively. Note that figure $2 \mathrm{a}$ also represents the time-evolution of the carrier density since the gain is assumed to linearly depend on $N$ in equations (1) and (2). Figure $2 c$ will be discussed later. As seen, the laser pulse is emitted after the laser gain has reached its maximum. This is the normal situation where only one pulse is emitted per period. The maximum-gain to threshold-gain ration is presently close to 1.2 . It rarely exceeds 1.5 under microwave modulation [41].

The instantaneous laser frequency evolution reported in figure $2 \mathrm{c}$ merits further comments. It is implicitely assumed that the laser is single-mode. In the semiconductor laser theory, the gain and refractive index variations are interrelated via a dimensionless parameter $\alpha$, known as the linewidth enhancement factor [19]: $\Delta n(t)=-\alpha(\lambda / 4 \pi) \Delta g(t)$. Since the frequency variations are also directly related to refractive index variations $(\Delta \nu(t)=-c \Delta n(t) / n \lambda)$, the laser frequency evolution can be described by :

$$
\Delta \nu(t)=\frac{\alpha c}{4 \pi n} \Delta g(t) \approx \frac{\alpha}{4 \pi}\left\{\Gamma A(1-\varepsilon S)\left(N-N_{0}\right)-1 / \tau_{\mathrm{p}}\right\},
$$

where the reference frequency is taken at the laser threshold.

From equation (3), a linear decrease of the gain is accompanied by a linear decrease of the laser frequency (downchirp). This situation is illustrated in figures $2 b$ and $2 c$. Pulses emitted from semiconductor lasers exhibit a quasi-linear downchirp. Non-linearities occur at the beginning and at the end of the pulse. Consequently, pulse compression can be realized by passing the downchirped pulses through a linearly dispersive medium such as an optical fiber [22-24]. Within the assumption of Gaussian pulses, the maximum compression rate is given by $\sqrt{1+\alpha^{2}} \approx \alpha[42]$.

Figure 3 shows the results of pulse compression experiments recently performed with a gain-switched DFB laser at $1.52 \mu \mathrm{m}$ and a $1 \mathrm{~km}$ dispersion-shifted fiber with a total dispersion 

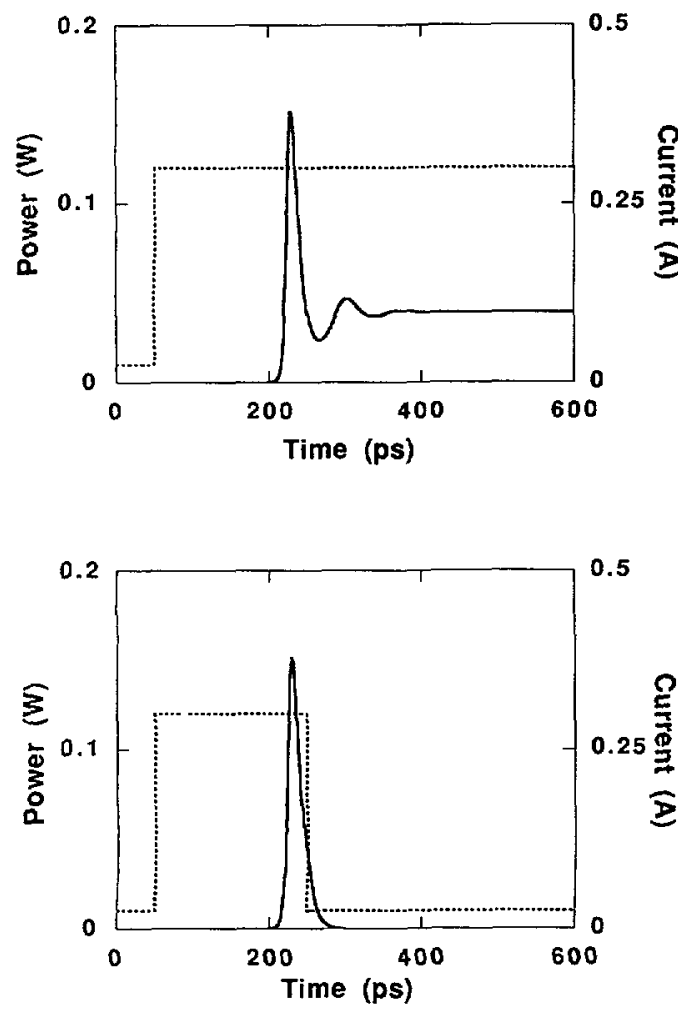

Fig. 1.
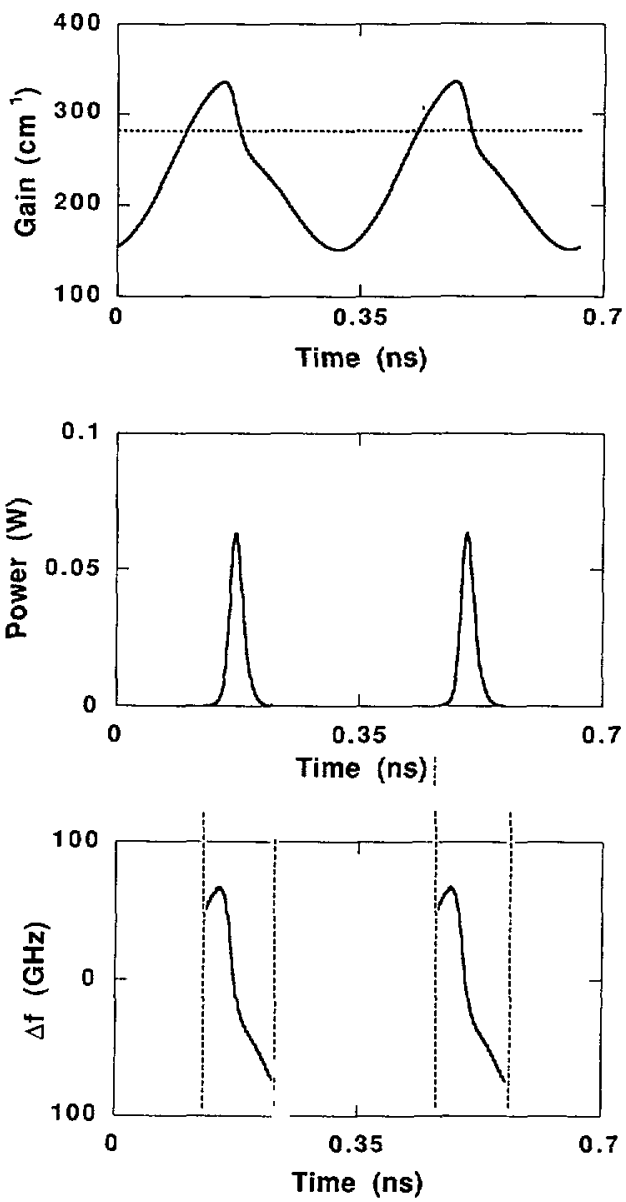

Fig. 2.

Fig. 1. - Laser responses under strong electrical excitation. a) single-step excitation, b) rectangular pulse excitation.

Fig. 2. - Time-evolutions of the gain a), output power b) and emitted frequency c) for a $1.5 \mu \mathrm{m}$ DFB laser under strong microwave modulation. Laser parameters are those reported in table I. The dc bias, modulation amplitude and modulation frequency are $I_{\mathrm{b}}=80 \mathrm{~mA}, I_{\mathrm{m}}=150 \mathrm{~mA}$ and $f=3 \mathrm{GHz}$, respectively. The dotted line in figure $2 \mathrm{a}$ represents the threshold gain.

of $-20 \mathrm{ps} / \mathrm{nm}$ [24]. Calculations from rate equations (1-3) are presented for comparison. The laser parameters (Tab. I) are determined from a set of measurements including the lightcurrent characteristics, the laser spectra under threshold, the optical measurement of the laser resonance frequency and the small-signal modulation characteristics at various biases [43]. Under strong microwave modulation $\left(f_{\mathrm{m}}=2.1 \mathrm{GHz}, I_{\mathrm{m}}=200 \mathrm{~mA}\right)$, the laser spectrum is measured to spread over $\sim 120 \mathrm{GHz}$ (Fig. 3a, bottom) and the pulsewidth before compression is deduced to be $\sim 18 \mathrm{ps}$ from autocorrelation measurements (FWHM, full-width halfmaximum). Calculations agree with experiments. The calculated spectrum (Fig. 3a, top) is $120 \mathrm{GHz}$ wide and exhibits a peak on the low-frequency side, the latter being related to the pulse tail of the downchirped pulse. The calculated pulse (Fig. 3b) is asymmetrical and has a width of $18 \mathrm{ps}$ (FWHM). Figure $3 \mathrm{c}$ shows the autocorrelation trace of the pulse after 
a)

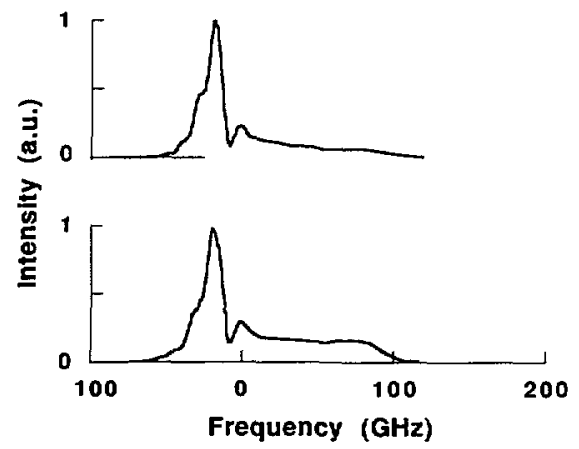

c)

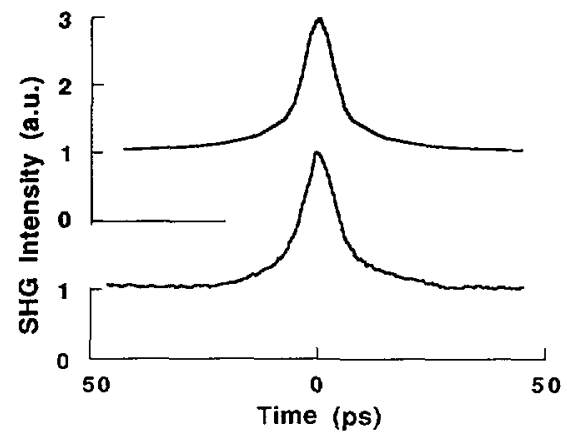

b)

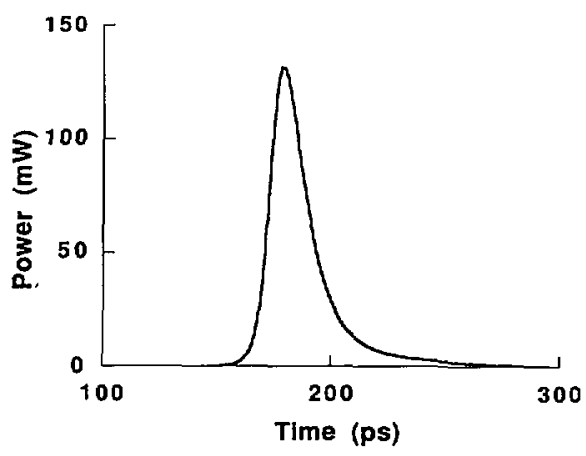

d)

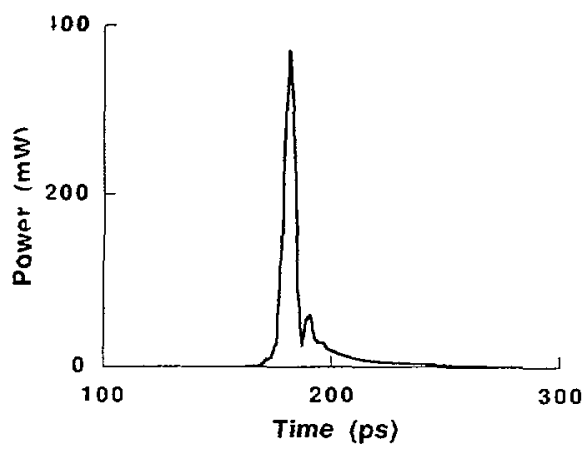

Fig. 3. - Fiber compression of gain-switched DFB laser pulses at $1.5 \mu \mathrm{m}$ (experiments and calculations). Laser parameters are those reported in table $\mathrm{I}$. The dc bias, modulation amplitude and modulation frequency are : $I_{b}=90 \mathrm{~mA}, I_{m}=200 \mathrm{~mA}$ and $f=2.1 \mathrm{GHz}$, respectively. The fiber dispersion is $-20 \mathrm{ps} / \mathrm{nm}$. a) laser spectrum - measurements (bottom) and calculations (top); b) calculated pulse before compression; c) autocorrelation trace of the compressed pulses - measurements (bottom) and calculations (top); d) calculated pulse after compression.

compression. Again, experiments (bottom curve) are well reproduced by calculations (top curve). The trace width is $8 \mathrm{ps}$ (FWHM). The existence of extended wings in the autocorrelation curve reveals some deviation from either a Gaussian or a secant hyperbolic shaped pulse. Actually, chirp non-linearities in the pulse before compression give rise to small side-lobes in the compressed pulse (Fig. 3d). Owing to the overall fit between calculations and experiments, a pulsewidth of 5.5 ps can be estimated after compression, which leads to a timebandwidth product $\Delta t . \Delta \nu$ of $\sim 0.6$. The compression rate $(\sim 3)$ is close to the value estimated for $\alpha(\sim 4)$. The peak power at the fiber output $(350 \mathrm{~mW})$ is more than one order of magnitude higher than the $\mathrm{CW}$ power delivered by the laser.

Still shorter pulses scaling from 2 to $4 \mathrm{ps}$ have been obtained by applying the same technique to different lasers [44]. Ultimate performances of gain-switching can be evaluated from an analytical approach of rate equations. Ignoring spontaneous emission and electrical pumping in equations (1) and (2) during gain-switching, analytical expressions are found for the total number of emitted photons, $\int S \mathrm{~d} t$, and the peak photon density, $S_{\max }$, respectively $[41,45]$. The pulsewidth is then deduced from these quantities. If gain non-linearities are taken into account, it is shown that for very high initial-inversion-ratios the minimum 
pulsewidth is given by :

$$
\Delta \tau_{1}=\tau_{p} \cdot \xi^{\left(\frac{\xi}{\xi-1}\right)}
$$

where $\tau_{\mathrm{p}}$ is the photon lifetime and $\xi=\varepsilon / A \tau_{\mathrm{p}}$. The linear and nonlinear gain coefficients, $A$ and $\varepsilon$, entering into $\xi$ are a priori material parameters while $\tau_{\mathrm{p}}$ is a cavity parameter. In the semiconductor laser terminology, $4 \pi^{2} \varepsilon / A$ is known as the $K$-factor and $f_{\mathrm{m}}=A / \pi \sqrt{2} \varepsilon$ is the maximum modulation bandwidth achievable with a given laser material [46].

The limits of equation (4) can be easily understood. In most cases, $\varepsilon / A$ is much larger than $\tau_{\mathrm{p}}$ (i.e., $\xi$ is much larger than 1 in Eq. (4)) so that the ultimate pulsewidth is close to $\varepsilon / A$. This value is obviously related to the smallest period of laser relaxation oscillations. The minimum $K$-value reported to date for long-wavelength lasers is $0.17 \mathrm{~ns}$ [47], which gives $\varepsilon / A \approx 4$ ps. Still smaller values are expected for short-wavelength lasers wich exhibit weaker gain-non-linearities. Complementing the gain-switching technique with fiber compression, sub-picosecond pulse generation thus appears to be feasible for semiconductor lasers with $\alpha$ larger than 5 .

A different limit is predicted for lasers with very long cavities since $\xi$ becomes less than 1 in equation (4). The minimum pulsewidth identifies in that case with the photon lifetime, $\tau_{\mathrm{p}}$. However, this result must be taken with care since propagation effects cannot be neglected so far in rate equation models. Indeed, the cavity roundtrip time, $\tau_{c}$, appears to be the true physical limit instead of $\tau_{p}$.

\section{Q-switching.}

Q-switching in semiconductor lasers is achieved by creating regions of saturable absorption in series with the gain medium (Fig. 4). Two main solutions are used. The first one is the implantation of ions through the diode facets [48-50]. The second one is the realization of multi-section laser geometries, where some of the sections are unbiased or reverse-biased [9, 51].

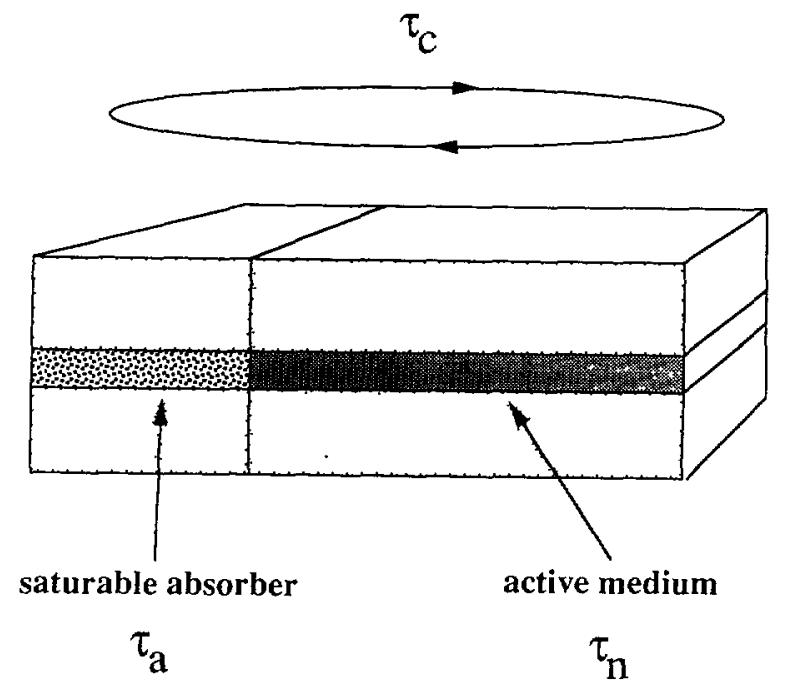

Fig. 4. - Basic scheme of a semiconductor laser with a saturable absorber. $\tau_{a}, \tau_{n}$ and $\tau_{c}$ are respectively the absorber relaxation time, the gain recovery time and the cavity roundtrip time. 
The mechanisms involved in diode laser Q-switching depend on the absorber relaxation time. The latter must be always shorter than the gain relaxation time in order to obtain a pulsed emission. However, it may be shorter or longer than the cavity roundtrip time, depending on the cavity length and the absorber material. The first situation may a priori lead to mode-locking. The laser dynamics in the second situation are similar to those of gainswitching.

Let us focus on the second situation. One difference with gain-switching is the possibility of generating optical pulses with a dc laser bias. In this pure Q-switching case, the pulse periodicity is fixed by the recovery time of the gain medium. Another difference is related to pulse shape. Q-switched pulses exhibit shorter tails than gain-switched pulses due to the absorber recovery at pulse end. However, the major interest of $Q$-switching stems from the achievement of very intense pulses under electrical pulse excitation. Absorber saturation effects are simultaneously combined with gain switching and the gain at maximum may be two times higher than the saturated cavity losses.

As for gain-switching, a good mathematical description of Q-switching is provided by rate equation models. Equations (1) and (2) of section 2 are accompanied by a third equation for the absorber population density $N_{\mathrm{a}}$.

$$
\frac{\mathrm{d} N_{\mathrm{a}}}{\mathrm{d} t}=-\frac{N_{\mathrm{a}}-N_{\mathrm{eq}}}{\tau_{\mathrm{a}}}-A_{\mathrm{a}} N_{\mathrm{a}} S .
$$

The two terms on the right-hand side of this equation represent the spontaneous carrier relaxation and the stimulated absorption, respectively. $N_{\mathrm{eq}}$ is the equilibrium absorber population in the absence of light, $\tau_{\mathrm{a}}$ is the absorber relaxation time and $A_{\mathrm{a}}$ is the absorber cross-section. The stimulated absorption term must be included as a loss term in equation (2). Approximate solutions of rate equations have been proposed in reference [49].

The precise description of phase modulation effects in the Q-switching regime is somewhat difficult due to the presence of the saturable absorber. The simplest approach is to assume an average value for the $\alpha$-parameter (Eq. (4) in Sect. 2). Under this assumption, the chirp amplitude during the pulse is proportional to the gain variations as well as to the electron density variations, a linear relation existing between $g$ and $N$. Since the number of emitted photons is approximately equal to the number of lost electrons, the total chirp amplitude is expected to be proportional to pulse energy.

Figure 5 shows the results of time-resolved spectroscopic experiments performed with Qswitched AlGaAs lasers of different pulse energies [52]. The lasers are operated in singlemode with the injection of a small $\mathrm{CW}$ monochromatic radiation in the cavity [53]. The pulse energy is varied by changing the ion-implantation conditions, i.e., by changing the spatial extension of the saturable absorber in the cavity. Electrical pumping is achieved with 2 ns pulses at a repetition rate of $40 \mathrm{MHz}$. Optical pulse measurements are made with a streak camera. As seen, the chirp amplitude is found to regularly increase with pulse energy. For the highest energy $(13 \mathrm{pJ})$, the chirp amplitude $(15 \AA)$ is about four times the cavity mode spacing.

Figure 6 shows results obtained in fiber compression experiments with the laser of highest energy. The pulsewidth measured by autocorrelation is $\sim 22 \mathrm{ps}$ before compression. The pulsewidth measured after compression is $\sim 1.3 \mathrm{ps}$, which agrees with previous measurements by streak camera [25]. This result is the best reported to date for a Q-switched semiconductor laser with electrical pumping. Beyond the performance, one may notice the extremely high value of the compression rate $(\sim 17)$, the latter being directly related to $\alpha$. Such a result agrees with semiconductor laser models which include gain non-linearities and predict an evolution of the form $\alpha=\alpha_{0} \sqrt{1+P / P_{\mathrm{s}}}$ for high-power lasers [54]. However, the 


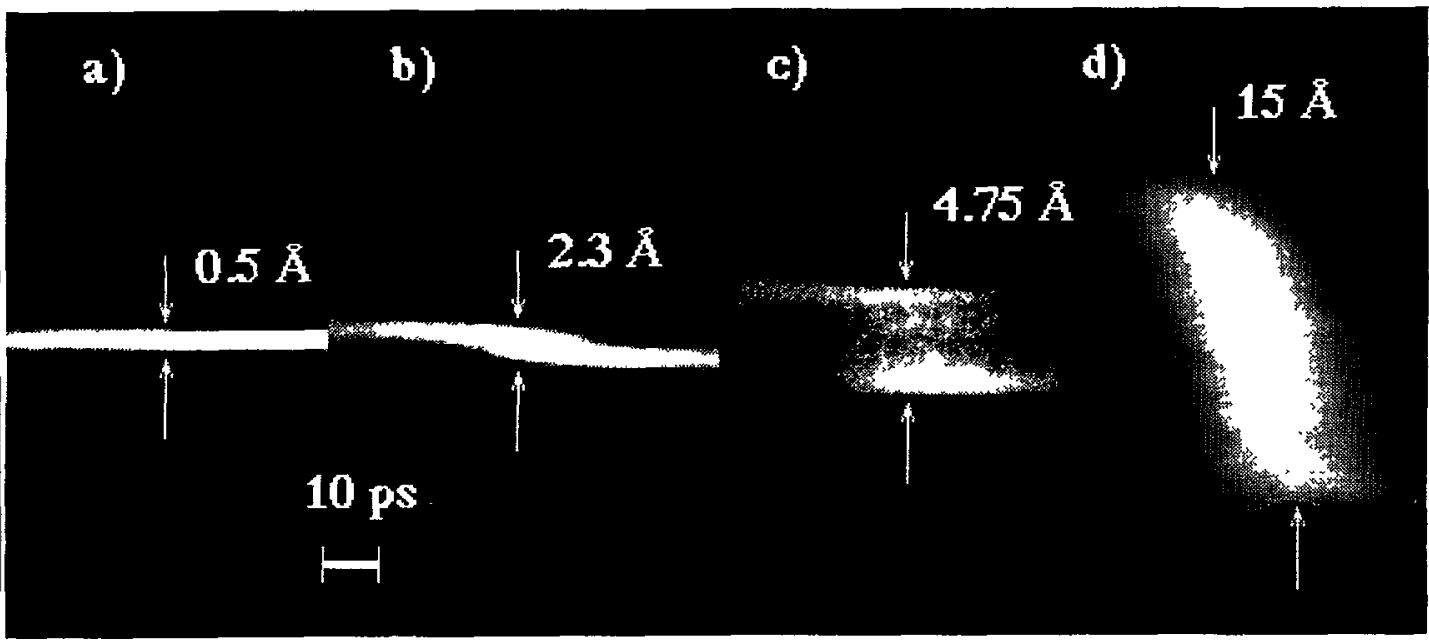

Fig. 5. - Time-resolved spectroscopic images of single-mode gain-/Q-switched laser pulses at $\sim 0.83 \mu \mathrm{m}$. a) HLP 1400 Hitachi laser without implantation $-E \approx 1.2 \mathrm{pJ}$; b) HLP 1400 Hitachi laser with $300 \mathrm{keV}$ proton implantation $-E \approx 2.5 \mathrm{pJ}$; c) HLP 1400 Hitachi laser with $11 \mathrm{MeV}$ oxygen implantation $-E \approx 5.8 \mathrm{pJ}$; d) gain-guided AlGaAs structure with $20 \mathrm{MeV}$ oxygen implantation $-E \approx 33 \mathrm{pJ}$.

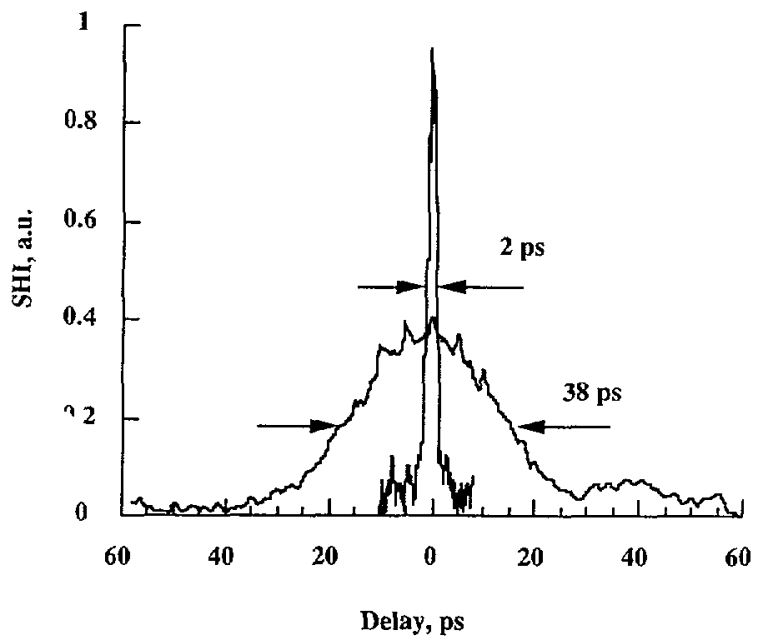

Fig. 6. - Second-harmonic autocorrelation traces of Q-switched pulses emitted by the gain-guided AlGaAs laser with $20 \mathrm{MeV}$ oxygen implantation. The trace width (FWHM) is $38 \mathrm{ps}$ without external compression. It becomes 2 ps after compression by a $\sim 200 \mathrm{~m}$ long fiber with a total dispersion of $-24 \mathrm{ps} / \mathrm{nm}$. A factor of 0.65 is used to deduce the laser pulsewidths in the text.

contribution of the saturable absorber should be explicitly taken into account for a more complete analysis.

The previous experimental illustrations clearly reveal the possibility of very large phasemodulation in single-mode Q-switched lasers. Due to high power levels resulting in strong laser non-linearities, the ultimate performances of Q-switching may be different from those previously established for gain-switched lasers. In principle, the ultimate limit of chirp 
amplitude is given by the gain-bandwidth. More realistically, chirp amplitudes of about $40 \AA$ can be expected at $0.8 \mu \mathrm{m}$ for a pulse energy of $\sim 30 \mathrm{pJ}$, which should correspond to $\sim 240 \mathrm{fs}$ pulses after compression.

\section{Mode-locking.}

Mode-locking is known as the most efficient technique to produce extremely short optical pulses from laser sources. For semiconductor lasers, mode-locking is effectively superior to the gain- and Q-switching techniques concerning the amplitude and timing jitter [30, 55] as well as the minimum width of the obtainable pulses $[16,17,29,38]$. However, it is also more complex. Among the large variety of effects observed in mode-locked semiconductor lasers, some of them still remain unexplained.

Basically, mode-locking is achieved by introducing a mechanism inside the laser cavity to cause the longitudinal modes to interact with one another thereby locking them in phase. One means is to modulate the laser gain at a frequency close to - or multiple of - the frequency spacing between the longitudinal modes. This technique is referred to as active mode-locking. However, for standard semiconductor laser devices, the cavity mode spacing is beyond $100 \mathrm{GHz}$ and it is very difficult to modulate the laser gain at these frequencies. Active modelocking requires the extension of the laser cavity. The most current solution consists of using an external reflector, the inner diode facet being antireflection (AR) coated (Fig. 7a). The second solution is to realize special devices such as monolithic extended-cavity lasers where the gain region is coupled to a low-loss passive waveguide $[31,56]$. In both cases, the minimum pulse repetition rate is fixed by the cavity mode spacing whereas the maximum repetition rate is limited by the laser modulation bandwidth.

The other means of achieving mode-locked laser pulses is to use a saturable absorber in the laser cavity. This technique which is referred to as passive mode-locking is best described in the time domain. As for Q-switching (see Sect. 3), the saturable absorber serves to sharpen up the pulse risetime while gain saturation effects combined with fast absorber recovery bring about an abrupt termination of the pulse [57-59]. The difference with Q-switching lies in the supplementary conditions imposed to the absorber relaxation time, $\tau_{\mathrm{a}}$ and to the cavity roundtrip time, $\tau_{c}$. The most favourable situation for passive mode-locking occurs when : $\tau_{c}>\tau_{n}>\tau_{a}, \tau_{n}$ being the recovery time of the gain medium. Since $\tau_{n}$ is of the order of $1 \mathrm{~ns}$ in semiconductor lasers, the previous condition can be only fulfilled if the cavity length exceeds a few centimeters. As for active mode-locking, the experimental solution is to use an external cavity (Fig. 7b). On the other hand, passive mode-locking may also occur when: $\tau_{\mathrm{n}} \geqslant \tau_{\mathrm{c}}>\tau_{\mathrm{a}}$. In principle, no external cavity is needed in that case, but very fast absorbers must be used. Mode-locked pulses were recently reported in two experiments without using an external cavity $[33,34]$ : the region of saturable absorption was realized by ion implantation in the first case [33] while a three-section laser with one reverse-biased section was used in the second case [34]. Interest of such configurations stems from the achievement of very high pulse repetition rates. The major drawback is the weak level of emitted peak power. As the gain medium cannot fully recover between two successive pulses, the population inversion rate always remains at a low value.

The complexity of the mode-locking process in semiconductor lasers mostly results from the use of an external cavity. Even if the residual reflectivity of the $A R$ coating is very low, say $10^{-4}$, the existence of the small laser diode cavity can never be ignored. In fact, the modelocked laser must be treated as a two-cavity system. This situation was first analyzed by Haus in $1980[60,61]$. Figure 8 gives a schematic representation in frequency-domain. Due to the presence of the diode cavity, the longitudinal modes of the extended cavity can be gathered into different clusters, each cluster being centered around one of the diode cavity resonances. 
a)

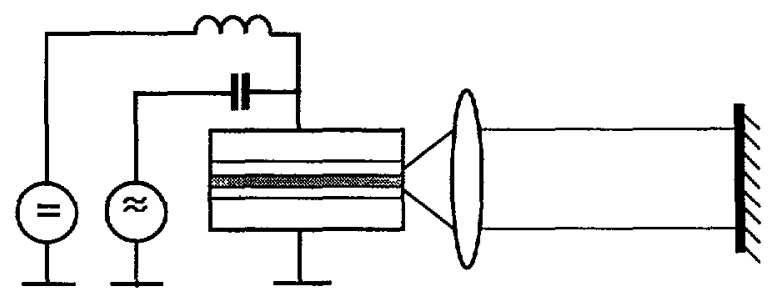

b)

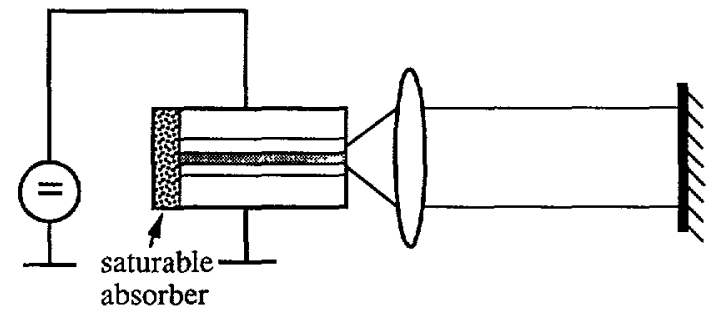

Fig. 7. - Basic schemes of a mode-locked semiconductor laser with extended cavity. a) active modelocking, b) passive mode-locking.

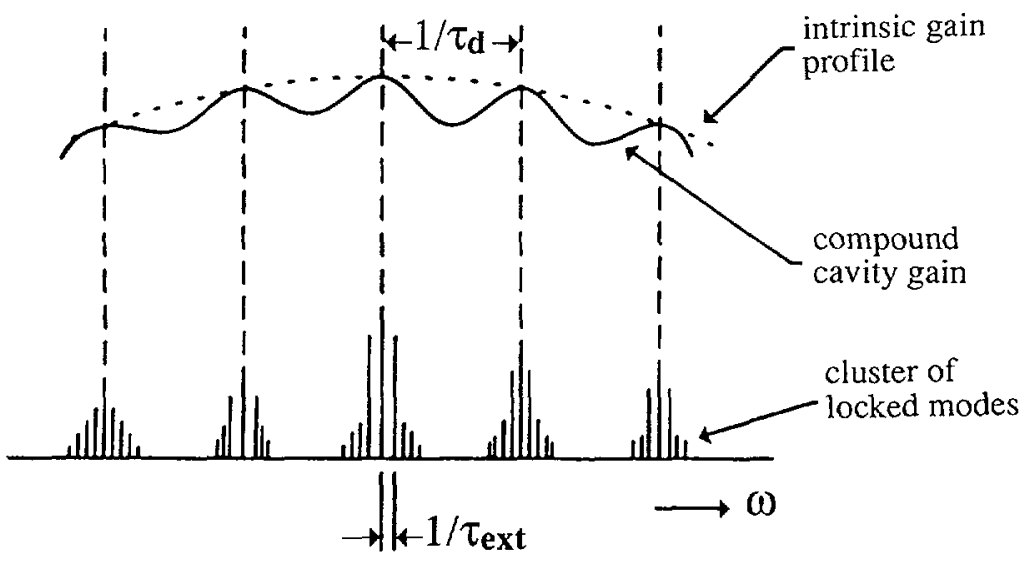

Fig. 8. - Mode-locking of a nonideally antireflection coated laser diode in an external cavity. The influence of the diode cavity resonances is simulated by a periodic gain profile (compound gain) [60]. $\tau_{\text {ext }}$ is the roundtrip time in the external cavity while $\tau_{\mathrm{d}}$ is the roundtrip time in the diode sub-cavity. Modes from neighboring clusters are not necessarily in phase with one another.

Using this simple picture, one imagines that the different modes of a given cluster can easily lock together. In contrast, modes from neighboring clusters are not necessarily in phase with one another. Mode-locking is then only partially realized and the emitted pulses are far to be transform-limited. The theoretical description proposed by Haus was based on a single master equation which gave the steady-state conditions for each longitudinal mode [60, 61]. The diode cavity resonances were simulated by using a periodic profile for the laser gain (see Fig. 8) and the pulsewidth limits were evaluated in this condition. 
Following the earlier work of Haus, mode-locking of semiconductor lasers in an external cavity was further analyzed in time-domain by several authors [30, 62-67]. Except for one case [63], analyses were directed at active mode-locking and numerical simulations were performed from rate equation models. For the long pulse regime $\left(\Delta \tau>\tau_{\mathrm{d}}\right)\left({ }^{*}\right)$, the basic equations were almost identical to those given in section 2 , except that a delayed feedback term of the form $k . S\left(t-\tau_{\text {ext }}\right)\left(^{* *}\right)$ was included in the photon density equation to account for the effect of the external cavity $[64,65]$. In agreement with experimental observations, laser instabilities where shown to occur when the roundtrip time in the external cavity was shorter than the period of recurrence for the current pulses [64]. For the short pulse regime $\left(\Delta \tau \ll \tau_{\mathrm{d}}\right)$, the rate equations were modified to include propagation effects in the diode cavity together with the boundary conditions at both the inner diode facet and the external reflector [30, 66-68]. In contrast, gain non-linearities were ignored for the sake of simplicity. The existence of a non-ideal $A R$ coating at the inner diode facet was found to result in the emission of laser pulse trains instead of regular mode-locked pulses, the time separation between successive pulses of a given pulse train being the roundtrip time in the diode cavity. In agreement with autocorrelation measurements [29,30], pulse trains consisting of three pulses were calculated for residual reflectivities as low as $10^{-4}$.

However, in spite of these theoretical fits, laser models still remain unadapted to predict the transition from the long pulse regime to the short pulse regime, i.e. from partial modelocking to full mode-locking. Experimentally, this transition abruptly occurs for a certain tuning of the external cavity and/or for a careful adjustment of the feedback coupling [29]. The behaviour is indifferently observed in passive and active mode-locking. In view of results reported in sections 2 and 3 , it is clear that chirping effects play an important role in semiconductor lasers. Improved models must then necessarily take them into account. Transverse effects may be also of some importance as revealed by recent experiments on other mode-locked systems such as Ti : Sapphire lasers [69, 70]. Experimental and theoretical investigations should be carried out in this direction for a better understanding of semiconductor laser dynamics.

Figure 9 shows recent results obtained by the authors on a passively mode-locked AlGaAs laser. The experimental scheme is close to that presented in figure $7 \mathrm{~b}$. A commercial HLP 1400 Hitachi laser diode is used. The saturable absorber is created by an $10 \mathrm{MeV}$ implantation of oxygen ions through the rear facet of the diode. The other facet is used for coupling to the external cavity. The absence of $A R$ coating in the present experiments places the facet reflectivity to a standard value of $\sim 30 \%$. The external cavity is $\sim 15 \mathrm{~cm}$ long and is comprised of an $A R$ coated microscope objective for beam collimation and an end-mirror with total reflection. Under normal conditions, the laser diode is dc biased. A modulation source can be additionally used to synchronize the pulses on an external reference clock.

The autocorrelation traces reported in figure $9 \mathrm{~b}$ well illustrate the previous discussion. It is clear that the mode-locked laser output is composed of pulse trains, the pulse spacing being the roundtrip time in the semiconductor amplifier $(\sim 8 \mathrm{ps})$. For most of the experimental conditions, the contrast between pulses is moderate : the modulation ratio is less than $60 \%$ (dotted curve). However, an abrupt change is observed for certain tunings of the external cavity and the modulation ratio increases up to $90 \%$ (solid curve). Simultaneously, the emitted spectrum is shifted towards shorter wavelengths and the spectral width is increased from 25 to $45 \AA$ (Fig. 9a). The minimum pulsewidth deduced in these conditions is $\sim 0.7 \mathrm{ps}$, which approximately corresponds to phase-locking of five clusters of modes

$\left.{ }^{*}\right)$ The pulsewidth, $\Delta \tau$, is larger than the roundtrip time in the diode cavity, $\tau_{\mathrm{d}}$.

${ }^{(*)} \tau_{\text {ext }}$ is the roundtrip time in the external cavity while $k$ determines the rate of optical feedback. 


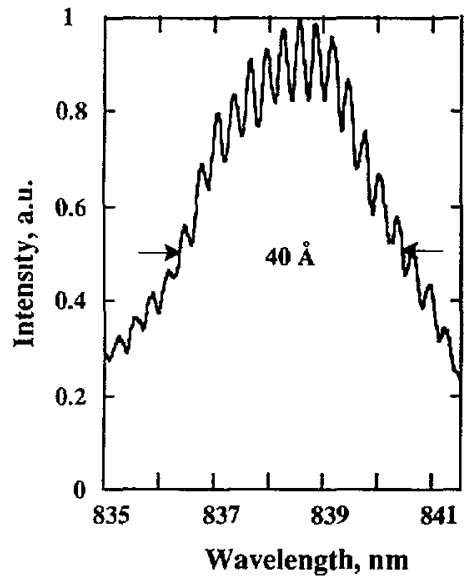

a)

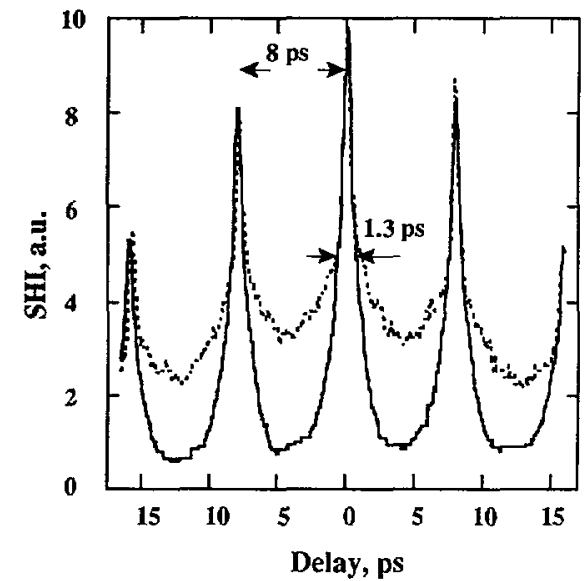

b)

Fig. 9. - Measured spectrum a) and autocorrelation traces b) of a passively mode-locked AlGaAs laser with a $15 \mathrm{~cm}$ long external cavity. Dotted curve and solid curve in b) respectively correspond to two different tunings of the external cavity (see text).

$(\Delta \lambda \approx 15 \AA)$. The average output power is $4 \mathrm{~mW}$, thereby indicating peak-powers around $1 \mathrm{~W}$. The performances are comparable to those previously published by Van der Ziel et al. [16].

As for Q-switching, the minimum pulsewidth obtainable by mode-locking is, in principle, determined by the inverse of the gain-bandwidth. This should a priori lead to femtosecond pulses ( $20 \mathrm{fs}$ ) since the gain-bandwidth typically scales from 20 to $60 \mathrm{~nm}$ in semiconductor lasers. On the other hand, some recent calculations from rate-equation models placed the limit just below $1 \mathrm{ps}$ in the case of active mode-locking [68]. Note that the authors finally questioned about the existence of pure « active mode-locking ». A more direct estimation can be made from the spectral width of the laser emission. In pulsed regime, laser diodes can emit up to 30 modes of reasonable amplitude (15 modes are obtained in Fig. 9). In the case of AlGaAs lasers at $0.8 \mu \mathrm{m}$, this approximately corresponds to a spectral width of $90 \AA$ and transform-limited pulses of $\sim 100 \mathrm{fs}$ can be envisaged.

\section{Prospects of further developments.}

Future developments can be expected in several directions. One important direction is to go towards femtosecond pulses. Research in this field is not only motivated by applications, but it is also of fundamental interest for a better knowledge of transient phenomena in semiconductor laser media. From discussions in sections 1, 2 and 3, it is clear that pulsewidths below $300 \mathrm{fs}$ already appear to be feasible. Recent results obtained by optical pumping confirm this assertion [40]. Mode-locking remains the most efficient technique in terms of short pulse generation, but optimized $A R$ coatings $\left(R<10^{-4}\right)$ are needed to eliminate parasitic effects in extended cavities. On the other hand, gain - or $Q$ - switching in vertical cavity surface emitting lasers (VCSEL) offers great promise [71]. Interest of VCSEL structures stems from the weak optical thickness of the gain medium $(<1 \mu \mathrm{m})$ leading in turn to very short cavity lengths $(<10 \mu \mathrm{m})$. Sub-femtosecond pulse-to-pulse timing jitters can be expected when spontaneous emission is localized in an active region of such a small thickness. Multiple-quantum-well VCSEL structures consisting of wells of different widths also appear 
to be an interesting solution for the realization of semiconductor lasers with wide gainbandwidth.

Another important evolution of picosecond semiconductor lasers is dictated by the rapid progress in the field of optical soliton transmission. Long-haul soliton transmission in fibers require Fourier transform-limited pulses of adjustable length at $1.55 \mu \mathrm{m}$. Perhaps, one of the most surprising challenge is the realization of coherent semiconductor laser pulses with durations of several tens of picoseconds and repetition rates scaling from 2.5 to $10 \mathrm{Gbit} / \mathrm{s}$. Actually, long pulses are needed to minimize pulse distorsions and dispersive wave radiation, both resulting from the use of lumped amplifiers regularly spaced along the optical link [72]. One technique for realizing such pulses is to use mode-locking in an external selective cavity [73], the pulsewidth being adjusted with spectral filtering in the cavity. However, solutions based on monolithic cavities are preferable for optical communication applications. Investigations are presently carried out in this direction. A first solution is to achieve mode-locking in very long monolithic cavities with integrated Bragg reflector [56]. A second solution is to extend the fiber compression technique to the case of gain-switched pulses of long duration. Single-mode lasers with small phase-amplitude coupling factors must be designed in order to limit the length of the dispersive fiber needed for compression. Strained-layer multiplequantum-well DFB lasers are good candidates [74].

Finally, semiconductor lasers able to deliver picosecond pulses of high energy are desired for a large number of applications. For instance, $\sim 200 \mathrm{pJ}$ pulses are typically required for optical triggering of fast electronic circuits. Up to now, the output energy of semiconductor lasers rarely exceeds $30 \mathrm{pJ}$ in the short-pulse regime and there is room for improvement. The maximum intensity which can propagate in the laser medium is determined by the two-photon absorption threshold. A typical value is $10^{9} \mathrm{~W} / \mathrm{cm}^{2}$. For a laser stripe of standard transverse dimensions $\left(\sim 0.2 \times 2.5 \mu \mathrm{m}^{2}\right)$, the maximum peak-power is then around $5 \mathrm{~W}$ and the maximum energy emitted in a 10 ps pulse is 50 pJ. Further improvements require the use of lasers with larger emitting surfaces. Several solutions can be considered: i) broad stripe lasers, ii) laser diode arrays, iii) lasers with stripe of large thickness (1-2 $\mu \mathrm{m})$, iv) vertical cavity surface emitting lasers (VCSEL). The two first solutions are commercially available, but transverse mode control is difficult. Solution iii) presents the advantage of output beams with weak transverse ellipticity. However, CW operation is excluded. Solution iv) a priori provides the largest emitting surfaces. A quick estimate indicates that peak powers up to $100 \mathrm{~W}$ should be obtained with solutions i), ii) or iii). Still one order of magnitude could be gained with solution iv).

\section{Concluding remarks.}

This paper has reviewed the current understanding of short pulse generation in semiconductor lasers. The different methods used to generate short pulses have been analyzed in details, some of the results presented for illustration being new. Possibilities of further improvements have been also discussed. However, some important aspects related for instance to lasers of low-dimensionality could not be specifically treated in this context. Actually, the development of low-dimensionality lasers as other recent developments is not necessarily motivated by applications to short pulse generation and high-speed modulation. Moreover, the laser performances are not related in a simple manner to the material parameters. For instance, it is now established that quantum well waveguide lasers do not exhibit better performances than bulk lasers of the same geometry. In contrast, progress acomplished in the growth of thin semiconductor layers allows the realization of new geometries such as vertical cavity lasers which are of great promise for high-power short-pulse generation. 


\section{Acknowledgment.}

The authors acknowledge the valuable and expert assistance of D. Pascal in the course of experiments.

\section{References}

[1] Mollenauer L. F., Nyman B. M., Neubelt M. J., Raybon G., Evangelides S. G., Demonstration of soliton transmission at $2.4 \mathrm{Gbit} / \mathrm{s}$ over $12000 \mathrm{~km}$, Electron Lett. 27 (1991) 178-179.

[2] NaKazawa M., Optical soliton communication using Erbium-doped fibre amplifier, 17th European Conference on Optical Communications ECOC' 91 (Paris 1991) Invited paper, pp. 150155.

[3] Iwatsuki K., Susuki K., Nishi S., Saruwatori M., Observation of $10 \mathrm{~Gb} / \mathrm{s}$ optical soliton over $10000 \mathrm{~km}$ using a gain-switched DFB-LD pulse source, 17th European Conference on Optical Communications ECOC' 91 (Paris 1991) Post-deadline paper, pp. 88-91.

[4] TAKADA A., SARUWATARI M., $100 \mathrm{Gbit} / \mathrm{s}$ optical signal generation by time-division multiplication of modulated and compressed pulses from gain-switched distributed feedback (DFB) laser diode, Electron Lett. 24 (1988) 1406-1408.

[5] TUCKER R. S., EISENSTEIN G., KOROTKY S. K., Optical time-division multiplexing for very highbit-rate systems, Conference on Laser and Electro-Optics CLEO 88 (Anaheim 1988), paper TUK5.

[6] Duvillaret L., Chusseau L., Lourtioz J.-M., Electro-optic Sampling of GaAs circuits using semiconductor lasers, 3rd European Conference on Electron and Optical Beam Testing of Integrated Circuits (Como 1991), Conf. Digest, pp. 246-253.

[7] Wiesenfeld J. M., Taylor A. J., Tucker R. S., EIsenstein G., BurRus C. A., Electro-optic sampling using injection lasers, SPIE Proc. 795 (1987) 339-344.

[8] Hall R. N., Fenner G. E., Kingsley J. D., Soltys T. J., Carlson R. O., Coherent light emission from GaAs junctions, Phys. Rev. Lett. 9 (1962) 366-368.

[9] LASHER G. J., Analysis of a proposed bistable laser, Solid State Electron. 7 (1964) 707-716.

[10] Kurnosov V. D., Magalyas V. I., Pleshkov A. A., Rivlin L. A., Trukhan V. G., TsveTKov V. V., Self modulation of emission from an injection semiconductor laser, JETP Lett. 4 (1966) 303-305.

[11] Morozov V. N., Nikitin V. V., Sheronov A. A., Self synchronization of modes in a GaAs semiconductor injection laser, JETP Lett. 7 (1968) 256-258.

[12] Zakharov Y. P., Kompanets I. N., Nikitin V. V., Semenov A. S., Synchronization of gallium arsenide laser light pulses, Sov. Phys. JETP 26 (1968) 895-896.

[13] HARrIS E. P., Spiking in current-modulated CW GaAs external cavity lasers, J. Appl. Phys. 42 (1971) 882-893.

[14] Glodge D., LeE T. P., Study of a self-pulsing GaAs laser by intensity correlation in Lithium Iodate, J. Appl. Phys. 42 (1971) 307-309.

[15] Ho P. T., Glasser L. A., Ippen E. P., Haus H. A., Picosecond pulse generation with a CW GaAlAs laser diode, Appl. Phys. Lett. 33 (1978) 241-242.

[16] Van der Ziel J. P., Tsang W. T., Logan R. A., Mikulyak R. M., Augustyniak W. M., T. P., Sub-picosecond pulses from passively mode-locked GaAs buried optical guide semiconductor lasers, Appl. Phys. Lett. 39 (1981) 525-527.

[17] Yoxoyama H., Ito H., INabA H., Generation of sub-picosecond coherent optical pulses by passive mode-locking of an AlGaAs semiconductor diode laser, Appl. Phys. Lett. 40 (1982) 105-107.

[18] Aspin G. J., Caroll J. E., Plumb R. G., The effect of cavity length on picosecond pulse generation with highly if modulated AlGaAs double heterostructure lasers, Appl. Phys. Lett. 39 (1981) 860-861. 
[19] HENRY C. H., Theory of the linewdith of semiconductor lasers, IEEE J. Quantum Electron. QE-18 (1982) 259-264.

[20] KOCH T. L., BOwERS J. E., Nature of wavelength chirping in directly modulated semiconductor lasers, Electron. Lett. 20 (1984) 1038-1040.

[21] Koch T. L., LINKE R. A., Effect of nonlinear gain reduction on semiconductor laser wavelength chirping, Appl. Phys. Lett. 48 (1981) 613-615.

[22] Takada A., Sugie T., Saruwatari M., Transform-limited $5.6 \mathrm{ps}$ optical pulse generation at $12 \mathrm{GHz}$ repetition rate from gain-switched distributed feedback laser diode by employing pulse compression technique, Electron. Lett. 22 (1986) 1347-1348.

[23] Takada A., Iwatsuki K., Saruwatari M., Picosecond laser diode pulse amplification up to $12 \mathrm{~W}$ by laser diode pumped erbium-doped fiber, IEEE Photon. Technol. Lett. 2 (1990) 122124.

[24] Chusseau L., XIE J. M., Duvillaret L., Lourtioz J.-M., Accard A., Hebert J.-P., Bandwidth-limited $0.3 \mathrm{~W}$ picosecond pulses (4 ps) from a $1.53 \mu \mathrm{m}$ microwave modulated DFB laser with fibre compression, Electron Lett. 26 (1990) 1085-1087.

[25] Stelmakh N., Lourtioz J.-M., BARTHELEMY A., Ultrashort pulse generation from a Q-switched AlGaAs laser with CW injection, Appl. Phys. Lett. 59 (1991) 624-626.

[26] Silberberg Y., SMITH P. W., Subpicosecond pulses from a mode-locked semiconductor laser, IEEE J. Quantum. Electron. QE-22 (1986) 759-761.

[27] Kuhl J., Serenyi M., Gobel E. O., Bandwidth-limited picosecond pulse generation in an actively mode-locked GaAs laser with intracavity chirp compensation, Opt. Lett. 12 (1987) 334-336.

[28] Eisenstein G., Tucker R. S., Koren U., KorotKy S. K., Actively mode-locking characteristics of InGaAsP-single mode fiber composite cavity lasers, IEEE J. Quantum Electron. QE-22 (1986) 142-148.

[29] Corzine S. W., Bowers J. E., Przybylek G., Koren U., Miller B. I., Soccholich C. E., Actively mode-locked GaInAsP laser with subpicosecond output, Appl. Phys. Lett. 52 (1987) 348-350.

[30] Bowers J. E., Morton P. A., Mar A., Corzine S. W., Actively mode-locked semiconductor lasers, IEEE J. Quantum Electron. QE-25 (1989) 1426-1439.

[31] Tucker R. S., Koren U., Raybon G., Miller B. I., Koch T. L., Eisenstein G., Active modelocked in a $1.5 \mu \mathrm{m}$ monolithic extended cavity laser, Electron. Lett. 25 (1989) 621-629.

[32] VASIL'ev P. P., Sergeev A. B., Generation of bandwidth-limited 2 ps pulses with $100 \mathrm{GHz}$ repetition rate from multisegmented injection laser, Electron. Lett. 25 (1989) 1049-1050.

[33] Zarrabi J. H., Portnoi E. L., Chelnokov A. V., Passive mode locking of a multistripe single quantum well GaAs laser diode with an intracavity saturable absorber, Appl. Phys. Lett. 59 (1991) 1526-1528.

[34] Chen Y. K., Wu M. C., Tanbun-Ek T., Logan R. A., Chin M. A., Subpicosecond monolithic colliding-pulse mode-locked multiple quantum well lasers, Appl. Phys. Lett. 58 (1991) 12531255.

[35] Tsuchry Y., Miwa M., Kolshi M., Direct observation of picosecond light pulses from a pulsecurrent pumped semiconductor laser, J. Appl. Phys. 53 (1982) 6671-6672.

[36] VAsil'ev P. P., Picosecond injection laser : a new technique for ultrafast Q-switching, IEEE $J$. Quantum Electron. 24 (1988) 2386-2391.

[37] PoRtnol E. L., Stel'makh N. M., Chelnokov A. V., Characteristics of heterostructure lasers with a saturable absorber fabricated by deep ion implantation, Sov. Tech. Phys. Lett. 15 (1989) 432-433.

[38] Delfyett P. J., Lee C. H., Florez L. T., Stoffel N. G., Gmitter T. J., Andreakis N. C., Alphonse G. A., Connolly J. C., Generation of sub-picosecond high-power optical pulses from a hybrid mode-locked semiconductor laser, Opt. Lett. 15 (1990) 1371-1373.

[39] NAKASAwa M., Susuki K., Yamada E., Femtosecond optical pulse generation using a distributed-feedback laser diode, Electron. Lett. 26 (1990) 2038-2040.

[40] Xiang W. H., Friberg S. R., Watanabe K., Machida S., Sakai Y., Iwamura H., YамAмото Y., Sub-100 femtosecond pulses from an external-cavity surface emitting 
InGaAs/InP multiple quantum well laser with soliton-effect compression, Appl. Phys. Lett. 59 (1991) 2076-2078.

[41] LAU K., Short pulse and high frequency signal generation in semiconductor lasers, J. Lightwave Technol. LT-7 (1989) 400-419.

[42] BuUs J., Propagation of chirped semiconductor laser pulses in monomode fibers, Appl. Opt. 24 (1985) 4196-4198.

[43] Hemery E., Chusseau L., Lourtioz J.-M., Dynamic behaviours of semiconductor lasers under strong sinusoidal current modulation: modeling and experiments at $1.3 \mu \mathrm{m}$, IEEE $J$. Quantum Electron. 26 (1990) 633-640.

[44] Cavelier M., Stelmakh N., Xie J.-M., Chusseau L., Lourtioz J.-M., Kazmierski C., Bouadma N., Picosecond ( $<2.5$ ps) wavelength-tunable $(\sim 20 \mathrm{~nm})$ semiconductor laser pulses with repetition rates up to $12 \mathrm{GHz}$, Electron. Lett. 28 (1992) 224-226.

[45] Wagner W. G., Lengyel B. A., Evolution of the giant pulse in a laser, J. Appl. Phys. 34 (1963) 2040-2046.

[46] TUCKER R. S., High-speed modulation of semiconductor lasers, J. Ligthwave Technol. LT-3 (1985) $1180-1192$.

[47] Nagarajan R., Fukushima T., Bowers J. E., Geel R. S., Coldren L., High-speed InGaAs/GaAs strained multiple quantum well lasers with low damping, Appl. Phys. Lett. 58 (1991) 2326-2328.

[48] Van der Ziel J. P., TSang W. T., Logan R. A., Augustyniak W. M., Pulsating output of separate confinement buried optical guide lasers due to the deliberate introduction of saturable loss, Appl. Phys. Lett. 39 (1981) 376-378.

[49] KuZNETSOV M., Pulsations of semiconductor lasers with a proton bombarded segment : welldeveloped pulsations, IEEE J. Quant. Electron. QE-21 (1985) 587-592.

[50] Alferov Zh. I., Zhularev A. B., Portnoi E. L., Stelmakh N. M., Picosecond pulses from Qswitched injection heterostructure lasers, Sov. Tech. Phys. Lett. 12 (1986) 452-453.

[51] TsANG D. Z., WALPOLE J. N., Q-switched semiconductor diode lasers, IEEE J. Quant. Electron. QE-19 (1983) 145-156.

[52] Stelmakh N., Lourtioz J.-M., Analysis of modal chirp in Q-switched semiconductor lasers, Electron. Lett. 27 (1991) 1856-1858.

[53] Stelmakh N., Lourtioz J.-M., Julien F. H., Injection-locking of Q-switched AlGaAs laser with fast saturable absorber, Electron. Lett. 27 (1991) 160-161.

[54] AGRawal G. P., Intensity dependence of the linewidth enhancement factor and its implication for semiconductor lasers, IEEE Photon. Technol. Lett. 1 (1989) 212-214.

[55] Derickson D. J., MAR A., Bowers J. E., Relative and absolute timing jitter in actively modelocked semiconductor lasers, 12th IEEE International Semiconductor Laser Conference (Davos 1990) Conf. Digest, pp. 138-139.

[56] Raybon G., Hansen P. B., Koren U., Miller B. I., Young M. G., Chien M., Burrus C. A., ALFERNESS R. C., A monolithic extended-cavity laser with an integrated Bragg reflector for active mode-locking at $8.3 \mathrm{GHz}, 17$ th European Conference on Optical Communications ECOC'91 (Paris 1991) Postdeadline paper, pp. 40-43.

[57] KRYukov P. G., LeToKhov V. S., Fluctuation mechanism of ultrashort pulse generation by laser with saturable absorber, IEEE J. Quantum Electron. QE-8 (1972) 766-782.

[58] Haus H. A., Theory of mode-locking with a slow saturable absorber, IEEE $I$. Quantum Electron. QE-11 (1975) 736-746.

[59] Haus H. A., Theory of mode-locking with a fast saturable absorber, J. Appl. Phys. 46 (1975) 30493058.

[60] Haus H. A., Theory of active mode-locking of a semiconductor laser in an external cavity, J. Appl. Phys. 51 (1980) 4042-4049.

[61] Haus H. A., Mode-locking of semiconductor laser diodes, Japan J. Appl. Phys. 20 (1981) 10071020.

[62] VAN DER ZIEL J. P., Active mode-locking of double heterostructure lasers in an external cavity, $J$. Appl. Phys. 52 (1981) 4435-4446. 
[63] Haus H. A., Silberberg Y., Theory of Mode-locking of a laser diode with a multiple-quantumwell structure, J. Opt. Soc. Am. B 2 (1985) 1237-1243.

[64] Garside B. K., KempF P., Influence of diode facet reflectivity on the dynamics of actively modelocked semiconductor lasers, Appl. Opt. 25 (1986) 3846-3849.

[65] Chesnoy J., Klein M. C., Chusseau L., Lourtioz J.-M., Period-doubling and periodquadrupling for an actively mode-locked laser diode with extended cavity, J. Appl. Phys. 67 (1990) 7615-7617.

[66] Demokan M. S., A model of a diode laser actively mode-locked by gain modulation, Int. $J$. Electron. 60 (1986) 67-85.

[67] Schell M., Weber A. G., Bóttcher E. H., Scholl E., Bimberg D., Theory of sub-picosecond pulse generation by active modelocking of a semiconductor laser amplifier in an external cavity : limit for the pulsewidth, IEEE J. Quantum Electron. QE-27 (1991) 402-409.

[68] Schell M., Weber A. G., Scholl E., Bimberg D., Fundamental limits of sub-ps pulse generation by active modelocking of a semiconductor lasers : the spectral gain width and the facet reflectivities, IEEE J. Quantum Electron. QE-27 (1991) 1661-1668.

[69] Negus D. K., Spinelli L., Goldblatt N., Feugnet G., Sub-100 fs pulse generation by Kerr Lens mode-locking InTi : $\mathrm{Al}_{2} \mathrm{O}_{3}$, Advanced Solid State Lasers Conference (Hilton Head 1991) Postdeadline paper PD4.

[70] Salin F., Squier J., Mourou G., Piché M., McCarthy N., Mode-locking of Ti : $\mathrm{Al}_{2} \mathrm{O}_{3}$ using self-focusing, Advanced Solid State Lasers Conference (Hilton Head 1991) Postdeadline paper PD5.

[71] Karin J. R., Melcer L. G., Nagarajan R., Bowers J. R., Corzine S. W., Morton P. A., GEELS R. S., COLDREN L. A., Generation of picosecond pulses with a gain-switched GaAs surface-emitting laser, Appl. Phys. Lett. 57 (1990) 963-965.

[72] Mollenauer L. F., Evengelides S. G., Haus H. A., Long-distance soliton propagation using lumped amplifiers and dispersion-shifted fiber, J. Lightwave Technol. 9 (1991) 194-197.

[73] Lundqvist S., ANDERsson T., ENG S. T., Generation of tunable single-mode picosecond pulses from an AlGaAs semiconductor laser with grating feedback, Appl. Phys. Lett. 43 (1983) 715 717.

[74] Kano F., Yoshikuni Y., Fukuda M., Yoshida J., Linewidth enhancement factor of $1.3 \mu \mathrm{m}$ InGaAsP/InP strained-layer multiple-quantum-well DFB lasers, IEEE Photon. Technol. Lett. 3 (1991) 877-879. 